\title{
The Association between Prolonged Occupational Exposure to Paraphenylene- diamine (Hair-dye) and Renal Impairment
}

\author{
Mohamed Hamdouk ${ }^{*}$, Mohamed Abdelraheem², Ahbab Taha ${ }^{1}$, David Cristina ${ }^{3}$, Chechepita Ionel ${ }^{3}$, \\ Ciocãlteu Alexandru ${ }^{3}$
}

1. Alsalam kidney centre, Khartoum, Sudan

2. Pediatric Nephrology Unit, Soba University hospital, Khartoum, Sudan

3. Saint John Emergency Hospital, Bucharest, Romania

\begin{abstract}
Introduction: Paraphenylenediamine (PPD) is widely used in hair dyes and cosmetic skin application. PPD intoxication following oral ingestion could be an important cause of ARF in Sudan, Morocco and the Indian Subcontinent. Repeated and prolonged exposure to PPD may also be associated with Chronic Kidney Disease (CKD).
\end{abstract}

Methods: This is a cross-sectional study that covered six conveniently-chosen hairdressing saloons in Khartoum, Sudan. Hairdressers with regular professional exposure to PPD were evaluated for the presence of renal impairment (serum creatinine $\geq 2 \mathrm{mg} / \mathrm{dl}$ ) and other markers of kidney damage.

Results: The study included seventy-two females with a mean age of $40 \pm 8$ years and a median duration of exposure to PPD of 6 years. Renal impairment, proteinuria and hematuria were observed in $14 \%, 26.4 \%$ and $41.1 \%$ of hair dressers, respectively. Hypertension, skin changes and bronchospasm were found in $19.4 \%, 38.9 \%$ and $22 \%$ of participants, respectively. Using pure forms of PPD significantly increased the risk of having elevated serum creatinine (OR 5.9; $\mathrm{P}=0.02)$ and proteinuria (OR 9.8; $\mathrm{P}=0.002$ ) compared to manufactured forms with lower concentrations. Each additional year of exposure to PPD significantly increased the risk of having elevated serum creatinine $(\mathrm{OR} 1.3 ; \mathrm{P}=0.01)$, proteinuria $(\mathrm{OR} 1.4 ; \mathrm{P}=$ 0.001 ) and hematuria (OR 1.1; $\mathrm{P}=0.04$ ).

Conclusion: In this group of hairdressers with regular exposure to PPD, we observed high prevalence of renal impairment, proteinuria and hematuria. These findings were significantly associated with the use of pure forms of PPD and longer duration of exposure.

\footnotetext{
* Corresponding author; Assalam Kidney Center, Khartoum, Sudan

E mail: hamdouk@hotmail.com
}

Keywords: Chronic Kidney Disease; Hair Dye; Paraphenylenediamine; Nephrotoxicity

\section{The authors declared no conflict of interest}

\section{Introduction}

Paraphenylenediamine PPD [C6 H4] is an aromatic amine not found in nature. It is a derivative of paranitroanaline that takes the form of white crystals when pure but rapidly turns to brown or black when exposed to air [1]. PPD is used in a variety of industrial processes and products: dyeing furs, photochemical processes, dark colored cosmetics, temporary tattoos, lithography plates, photocopying and printing inks, black rubber, oils, greases and gasoline. PPD is used in hair dye formulations to produce a variety of shades depending on the formulation; the exact concentration of PPD in different formulations is not known but the higher the concentration the deeper the color [2].

In Sudan, PPD is used by women to color their hair and skin after adding it to Henna, the extract of Lawasonia alba. Henna on its own is not toxic but needs to be applied to the skin two or three times for several hours to give the desired color (dark red or black). In contrast, the same result can be achieved after a single application of less than one hour by adding PPD to Henna.

Exposure to PPD has been linked with the development of acute kidney injury, systemic toxicity and allergic dermatitis [2]. PPD is a toxic allergen and well known to cause irritation and dermatitis when conveyed to the skin of susceptible people. These skin manifestations occur as a result of an allergic contact dermatitis, which generally manifest as an eczematous rash [3]. In a study of environmental and industrial exposure to PPD, thirtyeight beauticians were found to have occupational allergic contact dermatitis [3]. 
PPD is also known for its systemic severe toxicity when ingested in accidental, suicidal or homicidal attempts [4]. However, the systemic toxicity of PPD may occur through skin absorption [5]. There are some reported cases of severe systemic intoxication after topical application of pure PPD mixed with Henna or for dyeing hair [6].

Literature on the progression and/or development of chronic kidney disease (CKD) following PPD exposure is scanty. We hereby report our observation on chronic PPD toxicity.

\section{Methods}

This is a cross sectional study in a population with repeated professional exposure to PPD. Subjects were recruited from six conveniently chosen hairdressing saloons located in different parts of Khartoum, Sudan. All hairdressers "Hananat" who were responsible for the application of Henna-mixed PPD in these saloons were invited to participate in the study. Participants were evaluated collectively in Bahri Renal Center and Al-Salam Kidney Center in Khartoum and Saint John Emergency Hospital and Carol Davila University of Medicine and Pharmacy in Bucharest, Romania. Informed consent was obtained from participants and all information was kept confidentially. We interviewed each participant and completed a written data collection sheet including the following information: age, duration and frequency of exposure to PPD, physical examination and biochemical findings. Participants with history of hypertension, diabetes mellitus or known renal diseases were excluded from the study. Participants who had significant medical problems were counseled and referred for further management.

Hypertension was defined according to the guidelines of the International Society of Hypertension; i.e. $\geq 140 / 90$ [7]. Participants were screened for proteinuria using urine dipsticks. Subjects with positive urine dipsticks for proteins were further evaluated by quantitative assessment of protein in 24-hour urine collections. Hematuria was defined as more than $5 \mathrm{RBCs} / \mathrm{HPF}$ detected in a spot urine sample. Hemoglobin and renal parameters were also evaluated using an auto-analyzer machine. Abnormal findings were confirmed by repeated testing. Renal impairment was defined by serum creatinine level $\geq 2 \mathrm{mg} / \mathrm{dl}$. Renal biopsy was taken when clinically indicated. We also performed chemical analysis for the used different PPD preparations by high performance liquid chromatography (HPLC).

Results are summarized as the mean \pm standard deviation for continuous variables and proportions for categorical variables. Logistic regression analysis was used to evaluate potential risk factors.

\section{Results}

A total number of 72 hairdressers participated in the study. The mean age of the studied group was $40 \pm 8$ years (range 22-55 years). All of them were females, since hairdressing saloons in Sudan only employ females.

The median duration of exposure to PPD was six years (range 1-20 years). Exposure duration exceeded 10 years in $40 \%$ of the study population. HPLC of the used hair dye preparations showed the manufactured type to contain an average $10 \%$ of PPD, while the pure type contained $97 \%$ of PPD. The majority (58\%) of hairdressers used both the pure and manufactured forms; $25 \%$ used only manufactured preparations and $16.7 \%$ used only pure preparations.

Ten hairdressers $(14 \%)$ were found to have serum creatinine level $\geq 2 \mathrm{mg} / \mathrm{dl}$. Elevated serum creatinine was significantly associated with the use of pure forms of PPD and longer duration of exposure (Table 1). Nineteen participants $(26.4 \%)$ had albumiuria, which was found to be significantly associated with the use of pure forms of PPD and longer duration of exposure (Table 2). In repeated urinalysis there was persistent microscopic hematuria in 30 participants $(14.1 \%)$, and this was significantly associated with duration of exposure to PPD (Table 3).

Eight percutaneous renal biopsies were obtained from hairdressers in whom it was clinically justified. Indications included persistent proteinuria, hematuria or impaired renal function. Six of the eight biopsies showed signs of interstitial fibrosis. Three biopsies revealed glomerular lesions in the form of mesangial expansion, accentuated lobular architecture and hypercellularity. In three biopsies tubular atrophy was observed.

Twenty nine hairdressers (40.3\%) observed black colored urine during the working days. Hypertension was diagnosed in 14 subjects $(19.4 \%)$. None of them was previously known to be hypertensive. Twenty-two hairdressers $(28.8 \%)$ of the studied population had hemoglobin levels below $10 \mathrm{gm} / \mathrm{dl}$. Skin lesions, mainly irritant contact dermatitis, were observed in 28 participants (38.9\%). Nail changes were observed in $31.9 \%$, in the form of color change, skin peeling and dystrophic nails. Bronchitis, with bronchospasm, was diagnosed in $22 \%$ of the hairdressers. Eleven participants (15.3\%) had different neurological complications; including lower limb muscle weakness and diminished reflexes. Eight hairdressers (11.1\%) had ophthalmological manifestations in the form of conjunctival congestion, scleral pigmentation and blurring of vision. 
Table 1: risk factors for elevated serum creatinine in the study population (logistic regression analysis)

\begin{tabular}{llll}
\hline Risk factor & Odds ratio & P value & 95\% confidence interval \\
\hline Using pure forms of PPD* & 5.9 & 0.02 & $1.3-27$ \\
Exposure duration to PPD (years)* & 1.3 & 0.01 & $1.1-1.6$ \\
Age (years) & 0.9 & 0.2 & $0.8-1.0$
\end{tabular}

* statistically significant

Table 2: risk factors for proteinuria in the study population (logistic regression analysis)

\begin{tabular}{llll}
\hline Risk factor & Odds ratio & P value & 95\% confidence interval \\
\hline Using pure forms of PPD* & 9.8 & 0.02 & $2.4-40$ \\
Exposure duration to PPD (years)* & 1.4 & 0.01 & $1.1-1.6$ \\
Age (years) & 0.9 & 0.1 & $0.8-1.0$ \\
\hline
\end{tabular}

* statistically significant

Table 3: risk factors for hematuria in the study population (logistic regression analysis)

\begin{tabular}{llll}
\hline Risk factor & Odds ratio & P value & 95\% confidence interval \\
\hline Using pure forms of PPD & 1.2 & 0.7 & $0.5-2.5$ \\
Exposure duration to PPD (years)* & 1.1 & 0.01 & $1.0-1.3$ \\
Age (years) & 1.0 & 0.4 & $0.9-1.0$ \\
\hline
\end{tabular}

* statistically significant

\section{Discussion}

Acute systemic toxicity induced by PPD has been well studied in animals and humans. PPD toxicity was first reported by Nott and co-workers in 1924 [8]. Few years later, many authors reported the development of angiooedema, cardiotoxicity and nephrotoxicity [2, 3, 9]. It is well known that PPD toxicity occurs irrespective of the root of administration. Previous reports from the Sudan describe acute systemic toxicity with multiorgan involvement following oral administration [4]. Others reported acute systemic toxicity following dermal exposure and absorption [10]. However, reports on the chronic use of PPD are very limited.

One of the earliest signs of PPD toxicity is the development of dark colored urine. It has been previously reported following acute toxicity and dermal exposures [10]. In our study, $40 \%$ of subjects developed dark colored urine during their working days. This may indicate that repeated cumulative exposure to PPD can also change the color of urine. Dark colored urine has been attributed to myoglobinuria induced by PPD as well as to the excretion of PPD in urine [10].
In this study, we found high prevalence of hypertension (19.4\%). Proteinuria and hematuria were also highly prevalent, $26.4 \%$ and $41.1 \%$, respectively. These alarming figures are higher than previously reported results from screening studies. In a pilot population-based survey from the Sudan, including 273 participants, 30 participants $(11 \%)$ were shown to have Chronic Kidney Disease (CKD) and proteinuria was observed in only 16 subjects $(5.9 \%)$ [11].

In $28.8 \%$ of the hairdresser's anemia with hemoglobin less than $10 \mathrm{gm} / \mathrm{dl}$ was observed. No obvious causes were identified apart from the prolonged exposure to PPD. Their complete blood picture showed normocytic normochromic anemia. Reticulocyte count and other investigations were not done. It has been reported that PPD can cause aplastic anemia, but further studies are needed to explain the pathology of bone marrow toxicity [12].

Skin manifestations were commonly observed (38.9\%). It is well known that PPD handling is associated with skin lesions. In a study of skin exposure to permanent hair dye, 33 hairdressers were assessed using a previously 
evaluated hand rinse method. Hand rinse samples were collected from each hand before the start of hair dyeing, after application of the dye and after cutting the newly-dyed hair. The study concluded that hairdressers' skin is exposed to allergenic compounds during hair dyeing. Exposure occurs from dye application, from cutting newly-dyed hair and from background exposure. PPD was identified in percentages ranging from $10-30 \%$ of the studied samples [13].

The kidneys are particularly vulnerable to effects of toxic agents because of their high perfusion rate. Renal damage induced by chemicals is well known.

Renal lesions associated with PPD intoxication received much attention because most clinical investigators reported renal failure [3, 14]. In some cases where histopathological examination was performed renal injury was obvious. Acute tubular necrosis (ATN) was claimed to be the cause of ARF in some cases [14]. Others reported glomerulonephritis and interstitial nephritis in addition to ATN. Samples were obtained after different time intervals from the onset of exposure to PPD [15].

In this study, histopathological examination of eight kidney biopsies was done. Major changes suggesting CKD were seen in six biopsies. Interstitial fibrosis was seen in all six biopsies, co-existing with glomerular and tubular lesions in three of them. Three biopsies $(37.5 \%)$ showed glomerular lesions in the form of mesangial expansion, accentuated lobular architecture and hypercellularity. In three biopsies tubular atrophy was observed. Vascular lesion in the form of intimal thickening was observed in one biopsy, probably due to hypertension. The above findings suggest that PPD could be an important risk factor for the development of chronic kidney disease in the Sudan.

\section{Conclusion}

We conclude that regular exposure to PPD may be associated with various systemic complications, such as elevated serum creatinine, proteinuria and hematuria. Hypertension, anemia, dermatological and neurological manifestations were also common in the study group.

Our findings points to the potential role of PPD exposure in the development and/or progression of chronic kidney disease. Further experimental and clinical studies are required to confirm a causal relationship and to explain the underlying mechanisms by which PPD exerts its toxic effect on the kidneys. Public education programs and the collaboration of different health authorities are required to raise public awareness of PPD related hazards.

\section{References}

1. Grasseli JG; editor. CRC Atlas of Spectral Data and Physical Constants for Organic Compounds. Cleveland: Chemcial Rubger Co.; 1973. p. B-223.

2. Hamdouk MI, Abdelraheem MB, Taha AA, Benghanem M, De Broe ME. Paraphenylenediamine hair dye poisoning. In: De Broe ME, Porter GA, Bennett WM, Deray G; editors. Clinical Nephrotoxins: renal injury from drugs and chemicals. 3rd ed. New York: Springer. 2008. p. 671-9.

3. Nethercott JR, Macpherson M, Choi BC, Nixon B. Contact dermatitis in hairdressers. Contact Dermatitis.1986;14:73-9.

4. Yagi H, el Hind AM, Khalil SI. Acute poisoning from hair dye. East Afr Med J. 1991 Jun;68(6):404-11.

5. Mathur AK, Gupta BN, Narang S, Singh S, Mathur N, Sing A, Shukla LJ, Shanker R. Biochemical and histopathological changes following dermal exposure to paraphenylenediamine in guinea pigs. J Appl Toxicol. 1990 Oct;10(5):383-6.

6. D'Arcy PF. Fatalities with the use of a henna dye. Pharmacy international 1982;3:217-8.

7. 1999 World Health Organization-International Society of Hypertension Guidelines for the Management of Hypertension. Guidelines Subcommittee. J Hypertens 1999 Feb;17(2):151-83

8. Nott HW. Systemic poisoning by hair dye. Br Med J. 1924 Mar;1(3297):421-2.

9. Filali A, Semlali I, Ottaviano V, Fumari C, Corradini D, Soulamani R. A retrospective study of acute systemic poisoning of paraphenylenediamine (occidental takawt) in Morocco. Afr J Trad CAM. 2006;3(1):142-9.

10. Kiese M, Raucher E. The absorption of p-toluenediamine through human skin in hair dyeing. Toxicol Appl Pharmacol. 1968 Nov;13(3):325-31.

11. Hasan Abu-aisha, Elealeed AM Elhassan, Ammar H Khamis and Adil Abu Elmaali. Chronic kidney disease in police forces households in Khartoum, Sudan: pilot report. Arab Journal of Nephrology and Transplantation. 2009 May;2(2):21-6.

12. Hopkins JE, Manoharan A. Severe aplastic anaemia following the use of hair dye: report of two cases and review of literature. Postgrad Med J. 1985 November;61(721):1003-5.

13. Lind ML. Dermatitis in hairdressers as a problem in chemical control. Ann Occup Hyg. 2005 Aug;49(6):457-9. 
14. Suliman SM, Homeida M, Aboud OI. Paraphenylenediamine induced acute tubular necrosis following hair dye ingestion. Human Toxicol. 1983 Oct;2(4):633-5.
15. Hamdouk M. Paraphenylenediamine (Hair Dye) Acute Systemic toxicity [Master's Thesis]. [Sheffield] Sheffield Kidney Institute; 2001. PPD nephrotoxicity; p. 34-47. 


\section{Erratum to:}

The Association between Prolonged Occupational Exposure to Paraphenylenediamine (Hair-dye)

Mohamed Hamdouk, Mohamed Abdelraheem, Ahbab Taha, David Cristina, Chechepita Ionel, Ciocãlteu Alexandru Arab J Nephrol Transplant. 2011 Jan;4(1):21-5

One of authors names was misprinted, the authors list should be corrected as follows:

Mohamed Hamdouk, Mohamed Abdelraheem, Ahbab Taha, David Cristina, Ionel Alexandru Checherita, Ciocãlteu Alexandru 